Carbonate apatite structure of tooth means more or less a close approximation to the biological reality: besides carbonate further elements are present: $\mathrm{F}, \mathrm{Cl}, \mathrm{SO}_{4} ; \mathrm{Na}$, $\mathrm{K}, \mathrm{Mg}$-also there is the close relation to the protein pattern-whose influences to the lattice combination is too complex to perceive an additive dependence on one of these ions. We should not go further now than stating that enamel crystals have the structure of an apatite which is partly substituted by carbonate, fluoride, chloride etc. and which crystallizes according to the type of the often tested hydroxyapatite.

I want to point out, however, that the mineral substance of the tooth is to be considered as a paracrystalline apatitic texture. This means that, besides the well ordered crystalline regions, heavily fanlty ordered and amorphous mineral ranges are present, which do not differ in their qualitative chemical composition but show fluctuations in their stoichiometric combinations. This paracrystalline structure is necessarily involved in the chemical conditions of the mechanism of the tooth mineralization.

Zusammenfassung. Der Aufbau der Zahnhartsubstanz ist im Verlaufe ihrer Bildung durch mehrere Faktoren u.a. Begleitionen, Texturvorlage (Proteinmatrix) - bestimmt. Der Einfluss des Carbonatgehalts wurde in diesem Zusammenhang besonders im Hinblick auf dessen kristallchemische Funktion bei der Zahnmineralisation (parakristalline Struktur des teilweise isomorph substituierten Apatits) untersucht.

H. NEWESELY

Forschungsgruppe fïr Mikromorphologie im Fritz-HaberInstitut (Max-Planck-Gesellschaft), Berlin-Dahlem

(Deutschland), 5. August 1963.

\section{Structure of Nepseudin. Synthesis of Dehydronepseudin}

Nepseudin has recently been isolated along with neotenone, dolineone, and pachyrrhizin from the root of the leguminous plant Neorautanenia pseudopachyrrhiza ${ }^{1}$. As a result of degradative experiments, nepseudin has been shown to be the furanoisoflavanone (I) similar to neotenone but with a unique $2^{\prime}, 3^{\prime}, 4^{\prime}$-trimethoxylated ring A replacing the normal $2^{\prime}, 4^{\prime}, 5^{\prime}$-oxygenation pattern. On dehydrogenation with manganese dioxide, (I) has been converted into dehydronepseudin, the furanoisoflavone (II). We wish to report the total synthesis of (II) confirming the proposed structures.

Hoesch condensation of 6-hydroxy-2,3-dihydrobenzo[b] furan ${ }^{2}$ with 2,3,4-trimethoxybenzyl cyanide ${ }^{3}$ yielded 6-hydroxy-5-(2,3,4-trimethoxyphenylacetyl)-2, 3-dihydrobenzo[b] furan (III, m.p. $129^{\circ} \sim 130^{\circ}$, IR $1637 \mathrm{~cm}^{-1}$ (Nujol) (CO). Found: $\mathrm{C}, 66.37 ; \mathrm{H}, 5.99 . \mathrm{C}_{19} \mathrm{H}_{20} \mathrm{O}_{6}$ requires: C, $66.27 ; \mathrm{H}, 5.85 \%$ ). Treatment of (III) with ethyl ortho-<smiles>COc1ccc(C2COc3cc4occc4cc3C2=O)cc1</smiles>

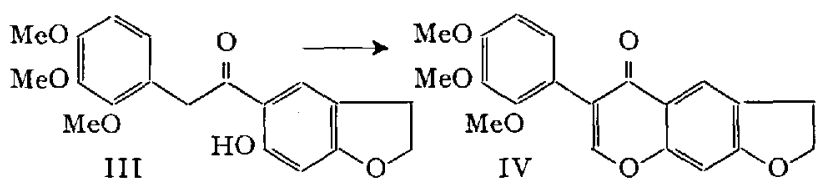

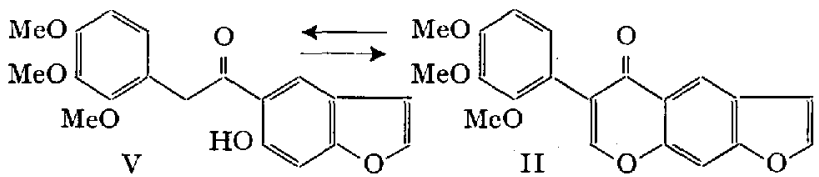

formate-pyridine-piperidine gave the dihydro compound (IV m. p. $143^{\circ} \sim 144^{\circ}$, IR $1628 \mathrm{~cm}^{-1}$ (Nujol) (CO). Found: $\mathrm{C}, 67.87 ; \mathrm{H}, 5.22 . \mathrm{C}_{20} \mathrm{H}_{18} \mathrm{O}_{8}$ requires: $\mathrm{C}, 67.79$; $\mathrm{H}, 5.12 \%$ ) of (II). On dehydrogenation with N-bromosuccinimide (IV) afforded dehydronepseudin (II, m.p. $164^{\circ} \sim 165^{\circ}$, IR $1649 \mathrm{~cm}^{-1}$ (Nujol) (CO), UV $\lambda_{\max }^{\text {EtOH }} \mathrm{m} \mu$ $(\log \varepsilon) ; 244$ (4.59), 324 (3.86). Found: C, 67.96; H, 4.61. $\mathrm{C}_{20} \mathrm{H}_{18} \mathrm{O}_{6}$ requires: C, 68.18; $\mathrm{H}, 4.58 \%$ )(lit. ${ }^{1}$, m.p. $158^{\circ}$ $\sim 160^{\circ}$, IR $1647 \mathrm{~cm}^{-1}$ (Nujol) (CO), UV $\lambda_{\max }^{\text {EtoH }} \mathrm{m} \mu(\log \varepsilon)$; 245 (4.64), $325(3.92)$ ). On alkaline hydrolysis (II) gave 6-hydroxy - 5- (2, 3, 4-trimethoxyphenylacetyl)benzo[b]furan ${ }^{1}$ (V, m.p. $118^{\circ}$, IR $1639 \mathrm{~cm}^{-1}$ (Nujol) (CO), UV $\lambda_{\max }^{\mathrm{EtOH}} \mathrm{m} \mu(\log \varepsilon) ; 236(4.76), 258.5(4.07), 272.5$ (3.97), 345 (3.71). Found: $\mathrm{C}, 66.69 ; \mathrm{H}, 5.40, \mathrm{C}_{18} \mathrm{H}_{18} \mathrm{O}_{6}$ requires: $\mathrm{C}$, $66.66 ; \mathrm{H}, 5.30 \%$ ) (lit. ${ }^{1}$, m.p. $118^{\circ} \sim 119^{\circ}$, IR $1645 \mathrm{~cm}^{-1}$ (Nujol) (CO), UV $\lambda_{\max }^{\mathrm{EtOH}} \mathrm{m} \mu(\log \varepsilon) ; 235$ (4.66), 258 (4.00), $270(3.86), 345(3.60))$. The parent isoflavone (II) could be reconstructed by treating (V) with ethyl orthoformatepyridine-piperidine. On the basis of these syntheses the structure of $(\mathrm{I})$ for nepseudin would be reasonably explained.

Zusammenfassung. Die Konstitution (I) für Nepseudin wird durch die Synthese des Dehydronepseudins (II), das aus 6-Oxy-2,3-dihydrobenzo[b]furan und 2,3,4-Trimethoxyphenylessigsäurenitril in drei Reaktionsstufen erhalten wird, aufgeklärt.

K. Fukui and M. Nakayama

Department of Chemistry, Faculty of Science, University of Hiroshima, Japan, July 9, 1963.

1 L. Crombie and D. A. Whiting, Tetrahedron Letters No, 18 , 801 (1962); Chem. and Ind. 1962, 1946; J. chem. Soc. 1963, 1569.

2 J. S. H. Davies, P. A. McCrea, W. L. Norris, and G. R. Ramage, J. chem. Soc. $1950,3206$.

3 K. Fukui, M. Nakayama, and N. Eto, J. chem. Soc. Japan, pure chem. sec. (Nippon Kagaku Zasshi) 84, 752 (1963). 